\title{
RIGOROUS LOWER BOUNDS FOR EXTINCTION PROBABILITIES OF THE CONTACT PROCESS
}

\author{
AIDAN SUDBURY, ${ }^{*}$ Monash University
}

\begin{abstract}
Positive correlations for extinction probabilities in the contact process are used to obtain rigorous lower bounds.
\end{abstract}

Keywords: Extinction; infinite particle system; contact process

2000 Mathematics Subject Classification: Primary 60K35

\section{The contact process}

The contact process is one of the most frequently studied interacting particle systems. Among other things, it can be used to model the spatial spread of an infection. In $d$-dimensions the state space is $\{0,1\}^{Z^{d}}$. We write $\eta(x)=0$ to mean that the site $x$ is empty (i.e. uninfected) and $\eta(x)=1$ to mean that it is occupied (i.e. infected). The sites act independently with the changes $1 \rightarrow 0$ at rate 1 and $0 \rightarrow 1$ at rate $\lambda$ times the number of occupied neighbours. For a detailed treatment, see Liggett (1985, Chapter VI). In Sudbury (2001), rigorous lower bounds for the critical infection rate were found for the diffusive contact process. The method used a computer to find a suitable submartingale. In this paper, a computer is used to create a set of inequalities.

In general, the difficulty in treating interacting particle systems derives from the fact that equations for sets of sites with span $n$ depend on the states of the sites with span $n+1$ in one dimension, and more than that in higher dimensions. For example, designating the probability of extinction from the set of sites $\cdots 0000001010000 \cdots$ as (101), we have

$$
(101)=\frac{1}{2+4 \lambda}[\lambda(1101)+2 \lambda(111)+\lambda(1011)+2(1)],
$$

since the configuration 101 may change to 1101 at rate $\lambda$, or to $\cdots 00001000 \cdots$ at rate 1 , etc. Note that the latter configuration is designated (1.1) using the convention that we only give the configuration between the left-most and right-most occupied sites. There is no systematic way of solving these equations, because the system is infinite. Our treatment is very similar to that of Konno (1994), although in this note we emphasize extinction probabilities rather than occupation probabilities for the upper invariant measure. The new ingredient is the use of a computer to find bounds which are more accurate, because it is then possible to treat much longer strings of $0 \mathrm{~s}$ and $1 \mathrm{~s}$.

Received 22 December 2004; revision received 2 February 2005.

* Postal address: School of Mathematical Sciences, Monash University, PO Box 28M, VIC 3800, Australia.

Email address: aidan.sudbury@sci.monash.edu.au 
We aim to find lower bounds on the extinction probabilities by using the positive correlations between extinction probabilities. For example,

$$
(1001100110101)>(100110)(0110101)=(10011)(110101) .
$$

Lemma 1.1. If $s_{1}$ and $s_{2}$ are strings of $0 s$ and $1 s$ then $\left(s_{1} s_{2}\right) \geq\left(s_{1}\right)\left(s_{2}\right)$.

Proof. We follow the treatment in Konno (1994, Chapter 3). Defining $v_{\lambda}$ as the upper invariant measure, Konno showed that, for any finite subsets of $Z, A$, and $B$, we have

$$
\bar{\rho}_{\lambda}(A \cup B) \geq \bar{\rho}_{\lambda}(A) \bar{\rho}_{\lambda}(B),
$$

where $\bar{\rho}_{\lambda}(A)=v_{\lambda}(\eta: \eta(x)=0$ for all $x \in A)$. The well-known duality connecting the distributions at time $t$, starting from initial sets $Z$ and $A$, i.e.

$$
\mathrm{P}\left(\eta_{t}^{Z} \cap A=\varnothing\right)=\mathrm{P}\left(\eta_{t}^{A} \cap Z=\varnothing\right),
$$

implies that $\bar{\rho}_{\lambda}(A)$ is equal to the probability of extinction with initial set $A$.

We initially set the lower bounds on the extinction probabilities to be 0 . We then derive the equations iteratively and use Lemma 1.1 to ensure that all the values we get are lower bounds for the extinction probabilities. The first four equations are (1.1) and

$$
\begin{aligned}
(1)_{m+1} & =\frac{1}{1+2 \lambda}\left(1+2 \lambda(11)_{m}\right), \\
(11)_{m+1} & =\frac{1}{2+2 \lambda}\left(2(1)_{m}+2 \lambda(111)_{m}\right), \\
(111)_{m+1} & =\frac{1}{3+2 \lambda}\left(2(11)_{m}+(101)_{m}+2 \lambda(1111)_{m}\right),
\end{aligned}
$$

equivalent to Konno (1994, Lemma 3.2.6). If we were only going to solve for spans of maximum length 3 , then (1.4) would be replaced by

$$
(111)_{m+1}=\frac{1}{3+2 \lambda}\left(2(11)_{m}+(101)_{m}+2 \lambda(11)_{m}(11)_{m}\right) .
$$

It should be noted that the best bounds are found when $s_{1}$ and $s_{2}$ in Lemma 1.1 are as similar as possible. The use of Lemma 1.1 in (1.2)-(1.3) gives the following theorem.

Theorem 1.1. We have

$$
\text { (1) } \geq \frac{1+\lambda}{\lambda(1+2 \lambda)}, \quad(11) \geq \frac{1}{2 \lambda^{2}} .
$$

These are reproduced here only because they are simple, as better bounds of this type can be found in Konno (1994, Lemmas 3.3.5 and 3.3.9).

\section{Results}

The results in Table 1 are for a variety of initial sets, with the maximum span being 14 . Starting from a single site, 20000 simulations were performed to give an indication of how close the lower bounds are in that case. 
TABLE 1: Initial occupied sites.

\begin{tabular}{cccccccccc}
\hline$\lambda$ & Simulations & 1 & 11 & 111 & 101 & 11111 & 10001 & 11111111 & 10000001 \\
\hline 1.7 & 0.627 & 0.546 & 0.412 & 0.333 & 0.367 & 0.233 & 0.329 & 0.141 & 0.304 \\
1.8 & 0.504 & 0.481 & 0.337 & 0.257 & 0.291 & 0.163 & 0.256 & 0.086 & 0.237 \\
1.9 & 0.444 & 0.431 & 0.281 & 0.202 & 0.236 & 0.116 & 0.204 & 0.054 & 0.189 \\
2.0 & 0.400 & 0.391 & 0.238 & 0.162 & 0.195 & 0.085 & 0.167 & 0.035 & 0.155 \\
2.5 & 0.268 & 0.271 & 0.125 & 0.066 & 0.093 & 0.022 & 0.077 & 0.005 & 0.074 \\
3.0 & 0.206 & 0.210 & 0.078 & 0.034 & 0.054 & 0.008 & 0.045 & 0.001 & 0.044 \\
\hline
\end{tabular}

\section{References}

Konno, N. (1994). Phase Transitions of Interacting Particle Systems. World Scientific, Singapore.

Liggett, T. M. (1985). Interacting Particle Systems. Springer, New York.

Sudbury, A. W. (2001). Rigorous lower bounds for the critical infection rate in the diffusive contact process. J. Appl. Prob. 38, 1074-1078. 